\title{
Body of evidence
}

I confess. Despite having no doubts over the accuracy of the mathematical formulation of general relativity or its standing as the correct physical theory of gravity, nor any doubts over the soundness of the various mathematical analyses leading to the notion of black holes, I've always secretly doubted that such things really exist. This hasn't stopped me reading happily about the properties of mathematical black holes, spinning, charged or otherwise; about amazing relationships such as that between a black hole's entropy and surface area, or the bizarre black-hole physics that throws up such oddities as wormholes in space-time.

But inwardly, I've always suspected that all this is probably more mathematics than physics, more imaginative possibility than reality, lacking any connection with empirical facts. This, I now see, is really an indefensible prejudice reflecting only my own ignorance of how far astrophysical observations have raced forwards, bringing masses of empirical data to bear on the existence of black holes. I just haven't been keeping up.

True, we still lack a knock-down proof that at least one black hole definitely exists somewhere in our Universe. Direct proof as Reinhard Genzel, Frank Eisenhauer and Stefan Gillessen note in a forthcoming paper in Reviews of Modern Physics - would require empirical access to the gravitational potential near the object's event horizon, something that is impossible at the moment.

But indirect evidence is strong, especially for a black hole in our own Galaxy. A wealth of observational evidence, including measurements of the dynamical trajectories of stars near the Galactic Centre, point to the existence of a dominant, compact mass for which a black hole looks to be the only plausible candidate. The once exotic and hypothetical now seems to be the established.

Hardly news, I'm sure, for most physicists (who have been keeping up), but the details of the progress leading to this point are worth revisiting. As is so often the case, spectacular advances on a seemingly impossible problem have come through painstaking experimentation and observation.

Donald Lynden-Bell first proposed, in 1969, that most galaxies might have black holes at their centres. His idea followed the discovery of distant, luminous quasars and the surmise that only the accretion of matter onto something as massive as a central black hole could account for their extreme luminosities.

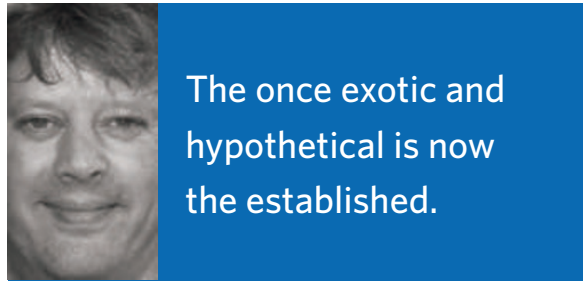

existence of an astrophysical black hole in our Galaxy. However, logically it establishes only the existence of a very massive compact object of unknown kind, which must be a black hole only if there's no other thing it might conceivably be. And there are possibilities.

For example, it could be an unusually dense cluster of faint stars or neutron stars, rocks, asteroids and other debris. However, there are tight constraints on the lifetime of such a cluster. At the known density of the object $-10^{16}$ solar masses per cubic parsec collisions and other processes should lead to either core collapse or evaporation (or both) on a timescale of only $10^{5}$ years. Most stars in the central cluster are far older than this; hence, it would be an extraordinary coincidence for the cluster to have formed so recently as to be still persisting.

Another possibility is the idea of a 'fermion ball' - a star of fermions, analogous to a neutron star, but made of, say, hypothetical massive neutrinos or other unknown massive fermions and supported against gravitational collapse by degeneracy pressure. Such an object could be very massive if the fermions making it up were very light. Yet the physical dimension of such an object also grows with decreasing fermion mass. Estimates suggest that a fermion ball massive enough to be the compact mass at the Galactic Centre would have a radius larger than the measured distance of closest approach of at least two of its orbiting stars. Hence, this doesn't seem plausible either.

There are still other exotic possibilities, but the black hole surmise does seem the most likely and natural. And it would certainly fit with lots of suggestive data from other galaxies. Evidence from nearly 50 nearby galaxies shows dark mass concentrations near their centres, and black holes seem the most likely interpretation there also.

The lesson, especially for me, is that it is easy to be prejudiced against something you don't know much about, especially if it seems exotic. Indeed, it is quite a natural response, although everything in the history of science shows the misguided wisdom of such an attitude, as the Universe really is more surprising than we ever expect it to be. The other lesson is that, with persistence, even the strangest of possibilities can be explored with that boring old engine of science - hard data.

\section{MARK BUCHANAN}

